\title{
Incidencia del consumo y marcas en la experiencia de bienestar subjetivo entre jóvenes: una exploración cualitativa
}

\section{Incidence of consumption and brands in subjective well-being experience among young people: a qualitative exploration}

\author{
Vargas Bianchi, L. ${ }^{1}$ \\ Recibido: 26-09-2018 - Aceptado: 30-01-2019 \\ DOI: https://doi.org/10.26441/RC18.1-2019-A9
}

\begin{abstract}
RESUMEN: Investigaciones señalan que el consumo se correlaciona con la experiencia de bienestar subjetivo. No obstante, es poca la literatura que trata sobre este fenómeno entre jóvenes. Este estudio exploratorio tiene por objeto conocer el modo en que estudiantes universitarios perciben que el consumo y las marcas intervienen sobre el bienestar subjetivo, e identificar los elementos que consideran en sus conductas de consumo destinadas a obtener bienestar. Se uso una metodología cualitativa de diseño fenomenológico, con estudiantes universitarios de la ciudad de Lima. El análisis siguió un proceso de codificación descriptiva. La experiencia de consumo y bienestar manifiesta ser un fenómeno relativo a cada persona, en particular su sentido de necesidad y marco valorativo de lo material, factores que parecen ser modulados por el entorno familiar. Los jóvenes encuentran en los actos de compra y las marcas un conducto para atender sus necesidades sociales, específicamente la pertenencia y aceptación en grupos.
\end{abstract}

Palabras clave: bienestar subjetivo; pertenencia; marcas; consumo; consumidor joven.

ABSTRACT: Research shows that consumption correlates with the experience of subjective wellbeing. However, there is little literature on this phenomenon among young people. This exploratory study aims to know how university students perceive that consumption and brands intervene on subjective well-being and identify the elements they consider in their consumption behaviors aimed at obtaining well-being. A qualitative methodology of phenomenological design was used, with university students from the city of Lima. The analysis followed a process of descriptive coding. The experience of consumption and well-being reveals itself as a phenomenon relative to each person, in particular their sense of need and value framework about material goods, factors that seem to be modulated by their family environment. Young people find in purchase practices and brands a conduit to meet their social needs, specifically belonging and acceptance in groups.

Key words: subjective well-being; belonging; brands, consumption; young consumers.

1 Lizardo Vargas Bianchi es Doctor en Comunicación Pública por la Universidad de Navarra, investigador y docente de Seminario de Investigación de Tesis en la Facultad de Comunicación de la Universidad Peruana de Ciencias Aplicadas. pcpulvar@upc.edu.pe, https://orcid.org/0000-0002-9027-7673 


\section{Introducción}

A lo largo de los años, los estudios en marketing se han centrado en las características funcionales de los productos, así como en el efecto de publicitar sus beneficios funcionales sobre la percepción del consumidor, y su influjo en las decisiones de compra. Sin embargo, el deseo de las personas por adquirir un bien no sólo se debe a sus funciones inherentes, como el sabor, su practicidad o el precio, sino también a otros aspectos vinculados con la compra. Por ejemplo, distintas investigaciones señalan que las experiencias de consumo y el ocio se correlacionan positivamente con la percepción de satisfacción general y bienestar de las personas (Tkach y Lyubomirsky, 2006; Kahneman, Krueger, Schkade, Schwarz y Stone, 2004). De acuerdo con los autores KimPrieto, Diener, Tamir, Scollon y Diener (2005), el bienestar subjetivo es un concepto amplio que se refiere a las diferentes formas de evaluar la propia vida, de acuerdo con las experiencias vividas y las propias emociones. Un amplio grupo de estudios sobre bienestar subjetivo contemplan la dimensión hedónica del bienestar, es decir, la referida a factores que inciden sobre la felicidad que las personas tienen respecto a su propia vida. (Fernández y GoñiGrandmontagne, 2011)

En cuanto al bienestar y el consumo, los autores Bettingen y Luedicke (2009) observan un vínculo entre el bienestar hedónico y las conductas de compra, al sostener que los individuos identifican el consumo de productos y marcas como un modo relevante y eficaz para lograr la felicidad. Estos autores, igual que Howell y Hill (2009) y Guevarra y Howell (2015), constatan que las creencias de las personas referidas al disfrute de bienes se relacionan con sus experiencias de bienestar. En una línea similar, estudios como los de Pirouz (2008) y de El Hedhli, Chebat y Sirgy (2013), señalan que los individuos reportan experimentar emociones de bienestar cuando participan en actividades de consumo, como ir de compras al centro comercial, o cuando deben seleccionar un producto entre varias opciones.

No obstante, existe poca literatura que explore las creencias y resultados anticipados respecto al consumo de bienes y su incidencia sobre el bienestar entre cohortes de adolescentes y jóvenes. Ante esta realidad, el presente estudio tiene por objeto conocer el modo en que estudiantes universitarios perciben que el consumo y las marcas intervienen sobre el estado de bienestar subjetivo, así como identificar los elementos que toman en consideración cuando realizan prácticas de consumo orientadas a experimentar bienestar. Para atender este objetivo se definió la siguiente pregunta de investigación:

PI: ¿Cómo comprenden los jóvenes que el consumo y las marcas participan en el estado de bienestar subjetivo?

$\mathrm{Al}$ atender la pregunta de investigación, este estudio profundiza en lo que un segmento de jóvenes con perfil similar entiende y proyecta sobre este aspecto del bienestar, desde su propia experiencia y la de sus pares. Asimismo, profundizar en las percepciones de consumo entre jóvenes aporta 
información sobre el consumidor en aspectos diferentes a la dimensión comercial. Si bien la literatura de marketing se centra en las transacciones comerciales, procurando hallar modos de facilitarlas, es relevante conocer otros aspectos de la conducta del consumidor. Contar con más información sobre la percepción entre consumo y bienestar entre los jóvenes, puede ser particularmente interesante en el entorno actual, donde consumidores de este grupo etario participan de modo activo en la creación de opiniones y percepciones asociadas con los productos que consumen. Por su parte, a los profesionales de marketing y la comunicación les es oportuno saber qué significa el bienestar con relación al consumo entre distintos segmentos de consumidores, con el objeto de mejorar la gestión de contenidos y en general las estrategias de promoción y comunicación de los productos.

Este trabajo tiene un alcance exploratorio sobre la percepción que jóvenes de un perfil determinado tienen respecto al consumo y el bienestar subjetivo. Tomar conocimiento de sus creencias y los factores que toman en consideración al procurar bienestar, añade nueva información al cuerpo de conocimiento para futuras elaboraciones teóricas y desarrollo de instrumentos de estudio. Para lograrlo, la metodología cualitativa es adecuada debido a que su objetivo no es generalizar el conocimiento referido a una población, sino comprender las experiencias de grupos específicos de individuos, o de un fenómeno particular, en su propio entorno.

\section{Bienestar Subjetivo}

Hacia finales del último milenio la psicología positiva se consolidó como un cuerpo integrado de conocimiento. Su origen se encuentra ligado al discurso que M. Seligman dirigió a la Asociación Americana de Psicología en 1997, cuando era presidente de esta organización, al motivar que los estudios en psicología no se centraran únicamente en las patologías o problemas de las personas, sino también en identificar los modos de incrementar su experiencia de felicidad (Linley, Harrington y Wood, 2006). En los años posteriores, Seligman, acompañado por un grupo de psicólogos (incluidos Mihaly Csikszentmihalyi, Ed Diener, Kathleen Jamieson, Chris Peterson y George Vaillant), establecieron las bases y el desarrollo temprano de este campo del conocimiento. De esa época en adelante, diversos autores publicaron obras y artículos especializados sobre psicología positiva y sus diversas vertientes. (Linley et al., 2006; Al Mandil, 2016).

Esta rama de la psicología se enfocó en desarrollar el conocimiento sobre la experiencia subjetiva positiva de las personas, y los rasgos individuales que pueden mejorar su calidad de vida, así como prevenir las patologías que surgen cuando el individuo experimenta su vida como estéril y carente de sentido (Seligman y Csikszentmihalyi, 2014). Puesto ahí el foco de interés, una de las principales áreas de estudio de la psicología positiva ha sido la felicidad o bienestar subjetivo de las personas.

Las investigaciones llevadas a cabo en las últimas décadas señalan que el 
bienestar subjetivo no es un concepto cerrado, sino que, por el contrario, se trata de una noción articulada y amplia. Distintos autores suscriben esta observación (Busseri \& Savada, 2011; Dolan, Peasgood y White, 2008; Oishi y Schimmack, 2010; Verma, 2017). De acuerdo con Diener (1994), Diener, Scollon y Lucas (1999) y Diener, Heintzelman, Kushlev, et. al. (2017), el bienestar subjetivo es la evaluación cognitiva que un individuo hace de la vida, en función a la presencia de emociones positivas y la falta de emociones negativas. Por su parte, la investigación realizada por Kim-Prieto, Diener, Tamir, Scollon y Diener (2005) añade que el bienestar subjetivo se refiere al modo articulado en que cada individuo evalúa su vida, basándose en sus experiencias, sus emociones, la satisfacción general o el afecto positivo que percibe están presentes en su biografía. Tov y Diener (2013) añaden que el bienestar subjeti- vo involucra las diversas formas en que las personas evalúan y experimentan sus vidas. Como se puede observar, las definiciones de estudios sobre bienestar subjetivo tienen elementos comunes que las acompañan de modo transversal. Un eje articulador entre ellas es la felicidad del individuo (Al Mandil, 2016), con lo cual un número de modelos de bienestar estructuran su concepto de bienestar desde una perspectiva hedónica, es decir de que el bienestar se obtiene evitando la incomodidad y procurando el mayor confort disponible (Brülde, 2014). Los autores Ryan y Deci (2001) añaden a esta afirmación cuando sostienen que la felicidad se encuentra representada por la agregación de los momentos hedónicos que experimenta el individuo. La Tabla 1 exhibe un resumen de los conceptos principales de las definiciones de bienestar subjetivo, y los autores quienes los postulan.

Tabla 1. Conceptos principales y autores de las definiciones de Bienestar Subjetivo (BS)

\begin{tabular}{|l|l|}
\hline Concepto central & Autores \\
\hline $\begin{array}{l}\text { BS considerado como un concepto compuesto, } \\
\text { articulado por distintos elementos de la persona y su } \\
\text { entorno. }\end{array}$ & $\begin{array}{l}\text { Busseri \& Savada, 2011; Dolan, Peasgood y } \\
\text { White, 2008; Oishi y Schimmack, 2010; } \\
\text { Verma, 2017. }\end{array}$ \\
\hline $\begin{array}{l}\text { BS como evaluación integral que el individuo hace de } \\
\text { su vida, en función a la presencia de emociones } \\
\text { positivas y la falta de emociones negativas }\end{array}$ & $\begin{array}{l}\text { Diener, 1994; Diener, Scollon y Lucas, } \\
\text { 2009; Diener, Heintzelman, Kushlev, et. al., } \\
\text { 2017; Diener, Lucas y Oshi, 2002, } \\
\text { Kim-Prieto, Diener, Tamir, Scollon y Diener, } \\
\text { 2005. }\end{array}$ \\
\hline $\begin{array}{l}\text { BS son las diversas formas en que las personas } \\
\text { evalúan y experimentan sus vidas. }\end{array}$ & Tov y Diener, 2013. \\
\hline $\begin{array}{l}\text { BS es una hedónica, se obtiene evitando la } \\
\text { incomodidad y procurando el mayor confort } \\
\text { disponible. }\end{array}$ & Brülde, 2014; Ryan y Deci, 2001. \\
\hline
\end{tabular}


El sentido de bienestar subjetivo que se utiliza en el presente estudio es la de Diener, Lucas y Oshi (2002), autores que ofrecen una definición concisa e integrativa: el bienestar subjetivo es el resultado de las evaluaciones cognitivas y afectivas que realiza una persona respecto a su vida. Asimismo, se adopta la consideración de Diener, Scollon y Lucas (2009), quienes consideran al bienestar subjetivo como una realidad articulada, compuesta de distintos factores tanto cognitivos como afectivos. Esta composición articulada sostenida por los autores se presenta en la Figura 1.

Figura 1. Modelo jerárquico de bienestar subjetivo.

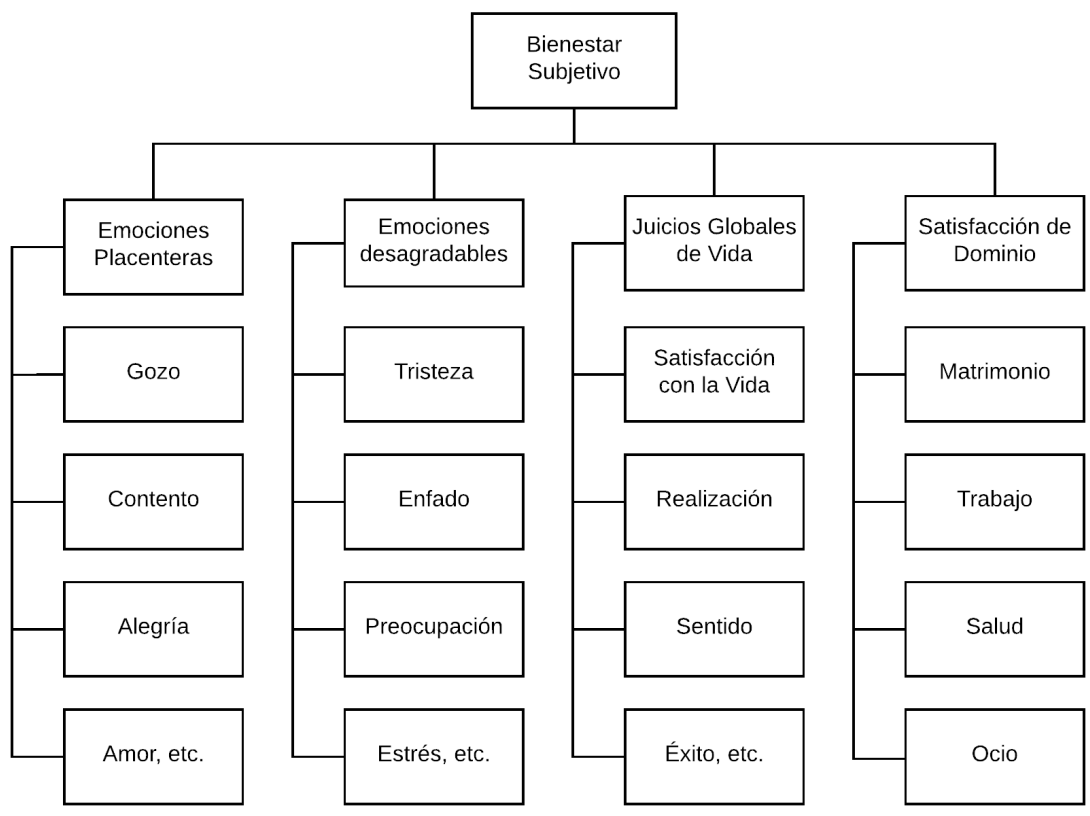

Fuente: Adaptado de Diener, et al. (2009)

Oishi, Suh y Lucas (1999) señalan que las múltiples diferencias individuales entre las personas son elementos que modulan la experiencia de bienestar. Estos autores sostienen que dos personas igualmente satisfechas con sus vidas y que, en promedio, experimentan la misma cantidad de emociones positivas y negativas, pueden haber llegado al estado de bienestar por distintas composiciones de eventos, estilos cognitivos, emociones o actitudes. Por ejemplo, una de estas hipotéticas personas puede sentirse satisfecha cuando derrota a sus amigos en una contienda deportiva, mientras que el otro experi- 
menta el mismo grado de satisfacción cuando recibe elogios de sus colegas por un logro profesional. A su vez, sostienen que la información sobre niveles medios del bienestar subjetivo de un grupo de personas no proporciona datos suficientes sobre las diferencias cualitativas individuales que dan lugar a su bienestar experimentado. De acuerdo con Oishi, Suh y Lucas esto se debe a la naturaleza articulada del bienestar tanto como al carácter subjetivo de las experiencias, realidad que resalta su naturaleza individual.

En cuanto a la medición del bienestar subjetivo, la mayor parte de los estudios realizados para identificarlo y analizarlo utilizan los datos provistos por las mismas personas. De acuerdo con Diener, Scollon y Lucas (1999), los individuos tienen la capacidad de auto-reportar su grado de bienestar utilizando su conciencia del presente y el recuerdo de las vivencias pasadas. Por su parte, Kahneman (1999), entre otros autores, consideran que es adecuado que el reporte del bienestar que realiza el sujeto contemple un espectro de momentos de su biografía, y no solo un presente muy reducido, de modo que le permita a la persona considerar en conjunto sus estados emocionales y de satisfacción (Kahneman y Tversky, 2003; Seligman y Czikszentmihalyi, 2000; Kahn y Juster, 2002). Con independencia del aspecto que se evalúe de las experiencias de bienestar, los trabajos orientados a medirlo siempre tratan de identificar los dominios, creencias y emociones, presentes en la vida de los individuos y que mayormente contribuyen a su bienestar. (Verma, 2017)
Respecto al bienestar y la realidad material de las personas, distintos trabajos constatan que algunos individuos perciben mayor bienestar si tienen más dinero disponible para consumir bienes o servicios (Chancellor y Lyubomirsky, 2014; Dittmar, Bond, Hurst y Kasser, 2014; Iyer y Muncy, 2016). Sin embargo, el efecto del consumo sobre el bienestar parece no ocurrir del mismo modo entre todas las personas. Investigaciones como las de Pirouz (2008) y Tov y Diener (2013) advierten que la influencia del ingreso económico sobre el bienestar subjetivo es mediada por el grado de deseos materiales de cada individuo, y entre sujetos que manifiestan mayor apego a estos actos, como comprar, buscar o consumir. Los deseos materiales y las prácticas de consumo son parte ordinaria de la vida de las personas, quienes invierten parte de su tiempo adquiriendo y utilizando bienes, y proyectando adquirir otros nuevos en el futuro (Diener y Biswas-Diener, 2002). El fenómeno se observa en análisis realizados con naciones industrializadas, así como diversas sociedades occidentales cuyos resultados muestran que las personas tienden a desear más de lo que poseen, y que habitualmente siempre tienen algo en mente que están deseando comprar, o sueñan con cosas que no les pertenecen (Schor, 1999). En cuanto a la disponibilidad monetaria, distintos autores explican que el dinero parece ser capaz de aliviar muchos de los males que enfrentan las personas cuando experimentan escasez de recursos, pero una vez que se alivian esos problemas, el dinero no parece mejorar, ni sostener 
por sí mismo, el bienestar subjetivo en el largo plazo (Kay y Jost 2003; Sengupta et al., 2012; Hoffmann y Lee, 2016; Dittmar, Bond, Hurst y Kasser, 2014). Como también señalan Diener y BiswasDiener (2002), el dinero y la adquisición de bienes materiales no parece asegurar la experiencia de bienestar de las personas.

Investigaciones como las de Iyer y Muncy (2016), o Chancellor y Lyubomirsky (2014), han reportado que, si bien los actos de consumo dan lugar a estados de satisfacción, con frecuencia estos estados son efímeros, pues la persona se acostumbra al estado de satisfacción alcanzado. Es decir que el bienestar obtenido en la compra, o el dinero disponible, eventualmente deja de constituir una experiencia de bienestar. Los autores sostienen que la vivencia positiva disminuye debido a la adaptación que el sujeto logra con el estado que adquiere. Cuando esto ocurre, la persona requiere de un nuevo acto de consumo para volver a experimentar el nivel de bienestar previo. Este fenómeno se conoce como adaptación hedónica, y se refiere a la tendencia general de las personas a regresar a un nivel establecido de felicidad. Por efecto de adaptación hedónica los sujetos se habitúan a la respuesta experimentada tras estímulo positivo o negativo, transcurrido el cual la percepción originada vuelve a su estado previo. La adaptación hedónica ha sido analizada en distintos trabajos (Armenta, Bao, Lyubomirsky y Sheldon, 2014; Buechel, Townsend y Moreau, 2018; Hovasapian y Levine, 2018). Este fenómeno (tam- bién conocido como cinta caminadora hedónica por referencia a la faja continua de las máquinas para hacer ejercicio), pone de manifiesto que la experiencia de bienestar subjetivo originada tras el consumo es susceptible de ser efímera, con lo cual se limita al momento en que la persona se acostumbra al bien del que dispone, o cuando este bien ya no provoca emociones positivas relevantes

Los conceptos de bienestar subjetivo, su vinculación con el consumo y las marcas, así como su dimensión referida a las realidades materiales y simbólicas, ofrecen un marco adecuado para analizar el modo en que los jóvenes comprenden que los bienes o marcas en general, pueden incidir sobre dicha variable.

\section{Metodología y procedimientos}

Para analizar el modo en que jóvenes perciben que el consumo incide sobre el bienestar subjetivo, e identificar las características que consideran en sus conductas de consumo orientadas al bienestar, se realizó una investigación cualitativa. Esta metodología permite explorar las experiencias y percepciones, tal como las manifiestan los individuos (Daymon y Holloway, 2010; Patton, 2002), en este caso sobre el bienestar, sus deseos y conductas de compra.

\subsection{Diseño del estudio}

La aproximación analítica de este estudio se realizó siguiendo un diseño fenomenológico. Mediante este enfoque cualitativo los investigadores se propo- 
nen comprender la experiencia de la vida humana, basándose en la información que el individuo ofrece en primera persona, y cuyos significados son compartidos entre otras personas, desde sus propias experiencias subjetivas. El diseño fenomenológico incorpora los elementos determinantes del mundo y de la vida de los individuos, sus entendimientos racionales y significados subjetivos, así como su acción social (Graneheim y Lundman, 2004), lo cual permite al investigador observar el fenómeno tal como ocurre en y desde la persona. El proceso de investigación cualitativa fenomenológica señala una sola fuente legítima de datos: la visión y experiencia de los participantes, los cuales se consideran como "hechos". Además, los participantes se seleccionan solo si han vivido la experiencia del tema de estudio. Por su parte, Titchen y Hobson (2011) establecen que el fenómeno puede ser estudiado directamente al explorar el conocimiento consciente de la persona, la que se esfuerza en otorgar sentido a sus experiencias, comprensiones y afectos. Los autores denominan esta aproximación fenomenológica como directa.

\subsection{Muestra}

Gentles, Ploeg y McKibbon, (2015) sostienen que el objetivo del muestreo en las investigaciones cualitativas es adquirir información útil para comprender la complejidad, la profundidad y el contexto que rodea a un fenómeno, en lugar de la generalización que procuran los estudios cuantitativos. Para la obtención de esta información útil, Palinkas et al. (2015) sostienen que las estrategias de muestreo cualitativo se deben orientar a seleccionar a las personas, o fuentes de datos, considerados 'ricos en información'. Por su parte, Yin (2011) amplía la idea al sostener que esa selección se puede realizar en función de la riqueza que el investigador anticipa que la fuente tendrá, así como la relevancia de la información con relación a la pregunta de la investigación.

En este estudio los participantes fueron estudiantes universitarios hombres y mujeres de la ciudad de Lima, todos ellos de carreras de ciencias sociales en una universidad privada, y pertenecientes a familias que cubren sus necesidades económicas. Se decidió investigar a este perfil específico de población joven para atender la pregunta de investigación por dos motivos. Primero, al ser estudiantes universitarios se aseguraba una capacidad razonable de abstracción y pensamiento conceptual, ambos útiles para describir sus experiencias de consumo y bienestar subjetivo. Segundo, al ser jóvenes que carecen de apuros económicos, se aseguró que los participantes tuvieran un repertorio suficiente de experiencias de consumo con productos y servicios que no estuvieran destinadas únicamente a atender necesidades básicas, sino también vinculadas con distintos niveles de hedonismo y bienestar. Por ejemplo, al contar con dinero disponible para actividades recreativas, los participantes tienen la posibilidad de orientar sus conductas de compra, y preferencias de marca, hacia aquellas que consideren brindarles mayor satisfacción. 
La selección de participantes para el estudio se realizó mediante un muestreo intencional. El muestreo intencional es la selección de casos ricos en información para el estudio en profundidad, donde se examinan aquellos de los que aprende sobre el tema central de la investigación (Patton, 2002). Los autores Palinkas et al. (2015) y Daymon y Holloway (2010) añaden que esta técnica de muestreo es ampliamente utilizada en la investigación cualitativa con el objeto de lograr la identificación de casos ricos en información relacionados con el fenómeno estudiado.

Para identificar participantes que cumplieran con ser fuentes de información relevante, se definieron una serie de criterios de inclusión (Daymon y Holloway, 2010). Solo aquellos estudiantes que cumplieran con la totalidad de estos criterios podrían formar parte del estudio. Los criterios de inclusión fueron: (i) ser estudiantes universitarios, (ii) tener entre 18 y 22 años, (iii) no ser estudiantes de publicidad o marketing, (iv) ser económicamente dependientes y (v) pertenecer a un nivel socioeconómico medio o superior.

La selección inicial se efectuó identificando participantes que cumplieron con los criterios de inclusión. Luego de seleccionar a los dos primeros participantes se siguió una estrategia de bola de nieve o muestras referidas, por la que los entrevistados derivaban a nuevos participantes como fuentes para estudio. Se utilizó esta segunda técnica debido a la dificultad para encontrar estudiantes con fechas y horarios disponibles para participar, y que además cumplieran con las condicio- nes definidas. Autores sostienen que la estrategia de bola de nieve es una de las prácticas del muestreo intencional. (Daymon y Holloway, 2010; Patton, 2002; Gentles, Ploeg y McKibbon, 2015)

El criterio generalmente aceptado en la investigación cualitativa para determinar cuándo se ha alcanzado un tamaño de muestra suficiente es la saturación de datos (Gentles, Ploeg y McKibbon, 2015; Morse, 1991). Fusch y Ness (2015) señalan que la saturación se identifica cuando el investigador llega al punto de no haber nuevos datos en sus fuentes o, en palabras de Palinkas et al. (2015), cuando ya no se adquiere información sustantiva nueva, o según Saunders et al. (2018) cuando la recolección o análisis de datos adicionales ya no es necesaria. Por su parte, Guest, Bunce y Johnson (2006) afirman que la saturación de datos se puede lograr con pocos casos como, por ejemplo, en tan solo seis entrevistas, y que lo importante no es el número sino la riqueza de información que el investigador ha obtenido de sus participantes o fuentes. En congruencia con la teoría expuesta, los participantes fueron seis estudiantes, pues la saturación de datos en este estudio se obtuvo en la sexta entrevista. Se identificó la saturación al percibir que ya no se obtenía información sustantiva nueva para codificar por parte de los participantes. (Fusch y Ness, 2015)

\subsection{Recolección de datos y procedimiento}

La obtención de datos se realizó mediante entrevistas en profundidad. Este instrumento es ampliamente utili- 
zado en los trabajos cualitativos, principalmente en aquellos que buscan comprender experiencias subjetivas, sentimientos o creencias de las personas (Crouch y McKenzie, 2006). Estos autores señalan que la entrevista en profundidad permite la conversación relativamente fluida con el participan- te, sin perder el enfoque de investigación para el cual se llevan a cabo. La entrevista en profundidad es generalmente utilizada en los estudios de diseño fenomenológico, debido al interés que cobra conocer el fenómeno tal como es vivido por otros sujetos. (Englander, 2012).

Tabla 2. Temas y preguntas guía para entrevistas en profundidad

\begin{tabular}{|l|l|}
\hline Tema & Preguntas semi-estructuradas \\
\hline Bienestar subjetivo & $\begin{array}{l}\text { ¿Qué entiendes por el bienestar de una persona? } \\
\text { ¿Cuáles son los componentes de [ese] bienestar? } \\
\text { ¿Relacionas el bienestar con la satisfacción sobre algún área de la } \\
\text { vida, o valoración de algún área de la vida? (Diener, et al., 2009) } \\
\text { ¿Relacionas el bienestar con emociones agradables / } \\
\text { desagradables? (Diener, et al., 2009) }\end{array}$ \\
\hline $\begin{array}{l}\text { Consumo/Prácticas de } \\
\text { consumo }\end{array}$ & $\begin{array}{l}\text { ¿Las personas, compran productos o marcas? } \\
\text { ¿Comprar un producto/marca determinada, influye en el bienestar? } \\
\text { ¿Qué clase de bienestar puede originar el consumo de un producto/ } \\
\text { servicio/marca? }\end{array}$ \\
\hline Comunicaciones de marca & $\begin{array}{l}\text { ¿Piensas que la publicidad tiene alguna función respecto a [las } \\
\text { prácticas o percepciones de consumo descritas en preguntas } \\
\text { previas]? } \\
\text { ¿Recuerdas algo/alguna publicidad que consideras que haya influido } \\
\text { tu consumo? } \\
\text { ¿Consumes alguna marca que no realice publicidad? }\end{array}$ \\
\hline
\end{tabular}

Fuente: Elaboración propia

Para utilizar esta técnica de recolección de datos, las preguntas de la entrevista deben estar estructuradas de modo que se puedan utilizar las mismas entre los distintos participantes, de lo contrario sería inviable lograr la saturación de datos (Fusch y Ness, 2015). Para ellos se elaboró una guía de indagación. Los temas referidos al bienestar subjetivo contenidos en la guía se basan en el modelo jerárquico de felicidad de Diener, Scollon y Lucas (2009) (ver la figura 1), y también sobre la afirmación de que los constructos para el estudio de bienestar subjetivo se pueden comprender como un continuo, con evaluaciones en tiempo real de la experiencia de las personas, de su estado emocional y emociones, así como sus evaluaciones generales de satisfacción en la vida (Stone y Mackie, 2013). Para atender la pregunta de investigación, la guía enmarcó tres temas: bienestar subjetivo, el consumo/prácticas de consumo, y las comunicaciones de marca. Cada tema contuvo tres preguntas semi-estructuradas de respuesta abierta. La Tabla 2 exhibe los temas y las 
preguntas utilizadas para guiar las entrevistas. Estas preguntas se formularon a todos los participantes, siempre en el mismo orden, para mantener la información recabada dentro del marco de la investigación (Fusch y Ness, 2015). No obstante, durante la entrevista el investigador podía añadir preguntas adicionales para ahondar en la información, o solicitar alguna precisión por parte del participante, procurando obtener datos útiles y valiosos (Palinkas et al., 2015; Gentles, Ploeg y McKibbon, 2015) relativos a la pregunta de investigación.

Las entrevistas fueron realizadas por el mismo investigador, dentro del campus universitario, en salas de estudio privadas, y de forma individual con cada participante. El investigador no había tenido ningún tipo de interacción previa con los participantes, y el contacto para coordinar las entrevistas se realizó mediante correo electrónico. Las entrevistas duraron entre 50 y $60 \mathrm{mi}$ nutos, y todas se registraron digitalmente. Posteriormente, las entrevistas fueron transcritas por un profesional en transcripción.

Respecto a los aspectos éticos de los procedimientos de investigación, se mantuvo el anonimato y la confidencialidad de la información de los participantes asignándoles un código de letras. Los participantes accedieron a participar de forma voluntaria, y se realizó un briefing, antes de que firmaran el consentimiento informado. En el briefing se les explicó que participarían en un estudio sobre la publicidad y las audiencias jóvenes. Finalizada la entrevis- ta se realizó el debrief a cada participante, ofreciendo mayor detalle sobre los objetivos del estudio. Ningún participante declinó continuar con el estudio ni requirió retirar la información proporcionada. No se ofreció a los participantes ningún incentivo ni compensación por su intervención.

\subsection{Análisis de la información}

Para realizar el análisis cualitativo, se siguió un proceso de codificación descriptiva, debido a que este método clasifica los datos de forma que permite explorar de modo organizado el fenómeno que busca conocer (Saldana, 2013). La codificación descriptiva permite identificar y transmitir, de forma directa, el fenómeno tal como es expresado por el participante, con lo cual es apropiada para atender el objeto de esta investigación.

Las unidades de análisis para realizar la codificación fueron definidas a prori, con base en los conceptos de bienestar subjetivo y el consumo. Las unidades fueron las siguientes: la Unidad de Codificación, es decir, la unidad de significado más pequeña que contiene información necesaria para atender la pregunta del estudio (Bengtsson, 2016), fue: Componente de significado claro (seme) en el texto. Por otra parte, la Unidad de Contexto establecida para facilitar las decisiones de codificación, fue definida como: "verbalización de la experiencia de bienestar relacionada con el consumo o una marca". El proceso de codificación inicial la realizó el investigador una vez finalizada la primera entrevista en profundidad, continuó 
conforme se llevaban a cabo las siguientes entrevistas, y continuó finalizado ese proceso. Para efectuar el análisis se utilizó el software QCAmap. (Fenzl y Philipp, 2013)

\section{Resultados}

Luego de llevar a cabo el proceso de codificación descriptiva con los datos obtenidos de las entrevistas, cinco categorías emergieron del análisis. El objetivo era conocer el modo en que estudiantes universitarios perciben que el consumo y las marcas intervienen sobre el bienestar subjetivo, e identificar los elementos considerados al realizar prácticas de consumo destinadas a obtener bienestar.

\section{Categoría 1: Necesidades cubiertas}

Esta categoría se refiere al sentido que los jóvenes otorgan al concepto de bienestar. Si bien los participantes no evidenciaron tener un significado unívoco del concepto, se hizo manifiesta la referencia común al hecho de tener las necesidades cubiertas como una condición basal para considerar la experiencia como de bienestar. El tipo, o naturaleza, de las necesidades parece importarles menos que percibirlas como adecuadamente atendidas. Los participantes señalaron una variedad de necesidades, desde las físicas hasta las de carácter moral para referirse, en todos los casos, a la relevancia de su satisfacción.

Por ejemplo, aludieron a necesidades tangibles e intangibles, como la alimentación o vivienda, tanto como la presencia de la familia y las amistades. Uno de los participantes lo expresa del siguiente modo: "es bienestar cuando tengo equilibrio emocional, físico, tener salud y comodidad". Otros dijeron: "es tener una familia, una casa donde llegar, tener siempre qué comer, (...) tener amigos, siempre contar con alguien más ante un problema", "poder cubrir todas las necesidades fisiológicas y las de autosuperación para lograr las cosas que se desean".

Un aspecto interesante entre las necesidades intangibles mencionadas es la referencia al sentido de uno mismo, y la necesidad de atender la propia estima. La atención a esta dimensión de la persona aparece como un concepto relevante en la experiencia del bienestar de los participantes. Uno de los estudiantes lo verbalizó del siguiente modo: "[bienestar es] sentirse bien con el entorno y consigo mismo (...) con lo que haces y con lo que realizarás a futuro". Eventualmente, esta persona vincula lo tangible con el sentido de sí, como un origen de las experiencias de bienestar: "Al sentir que tienes el poder de comprar algo mejor, te sientes mejor contigo mismo".

Las necesidades aparecen como realidades que subyacen en las ideas y conductas de las personas, y aparecen como condiciones de bienestar, al margen del tipo de necesidad o su grado de especificidad.

\section{Categoría 2: Aprendizaje en la familia}

Esta categoría describe la incidencia del entorno familiar sobre la constitución del sentido y la valoración que los jóvenes otorgan al consumo y las marcas. Es decir, el nivel de relevancia que conceden a estas realidades en sus 
vidas. Los participantes señalan que es su familia el espacio donde aprendieron del sentido del consumo y, también, los parámetros valorativos que en actualidad tienen hacia este concepto y las marcas.

Los participantes expresan que el entorno familiar ha guiado sus criterios de valoración hacia lo material, así como su sentido en la constitución de la identidad. Un estudiante lo expresa del siguiente modo: "depende mucho de la formación, de la manera en la que hayan criado tus papás (...) Si mis padres no me dieron a entender que las marcas son tan importantes, a mí no me va a importar".

La ordenación de los hábitos, así como la valoración a la realidad de consumo, se presenta vinculada con las conductas observadas y la formación adquirida en el hogar. Por ejemplo, la declaración "yo no me considero una persona consumista y eso también tiene que ver con mi familia", exhibe la incidencia del círculo familiar en la conformación del marco valorativo del individuo.

No obstante, un participante señala que las conductas y valoraciones aprendidas no son determinantes: "Tu familia te acostumbra hasta cierto punto, pero de ahí ya tú decides". El entrevistado rescata la posibilidad de ejercitar su libertad y desde ello definir la amplitud de lo aprendido. Es decir, que puede auto determinarse en cuanto a sus esquemas valorativos sobre aquello que compra, su valoración hacia las marcas, el materialismo u otros factores del consumo.

A pesar de que no es determinante, el influjo de lo aprendido en la familia se percibe como una realidad que pu- ede acompañar en el largo plazo, y de la cual no sería posible alejarse del todo. "Hay personas que hasta ahorran bastante para comprarse algo y es el círculo vicioso del consumismo. [¿̇Y eso acaba?] Siento que no, hasta que cuando ya esté mayor y sienta que ya haya tenido todo".

\section{Categoría 3: Relativo a cada uno}

Esta categoría describe el hecho de que la relevancia, e influencia, del consumo y marcas entre los jóvenes es parte de una dinámica personal. Es decir que cada individuo posee su propio estilo cognitivo y afectivo individual, así como su propia realidad económica. Como se ha visto, también sus marcos valorativos personales. Esta realidad da lugar a múltiples posibilidades de búsquedas y experiencia de bienestar en la compra de bienes.

Uno de los participantes lo ejemplifica de la siguiente manera: "Es muy variable, tengo amigos que cuando salen a comprar prefieren comprarse dos polos de tal marca, a solo un polo de una marca por el mismo precio, pero también tengo amigos que prefieren lo contrario".

Al dialogar sobre el bienestar y el consumo, algunos otorgan poca relevancia a la marca: "A mí no me importa mucho cuando me preguntan qué marca es lo que uso". Por otro lado, el carácter o forma de ser de cada uno incide también en la relevancia que le otorga al consumo o tenencia de marcas; por ejemplo: "Si una persona tiene mucha personalidad no le importa venir como sea con tal de estar en clase, pero a una persona que sí le afecta más el qué dirán tal vez sí le incomode más". 
La manera de buscar bienestar mediante el consumo de marcas se revela como una realidad relativa a cada individuo. Un estudiante lo expone: "Creo que un poco depende de qué te quieres comprar y cómo eso te va a ayudar". Es decir, la definición del modo en que un bien le otorgará el beneficio proyectado, se halla en función a factores personales, como los criterios de valoración del sujeto, los riesgos que percibe, las presiones de grupo, o su identidad personal.

\section{Categoría 4: Conciencia de lo temporal}

Esta categoría describe la conciencia que tienen los jóvenes de que el consumo de lo material, destinado al originar bienestar, acarrea la posibilidad de ser pasajero o efímero. Los participantes manifiestan haber contactado con la conciencia de que algunas realidades son temporales, en cuanto que no son un conducto permanente de bienestar o satisfacción.

Uno de los participantes refiere esta conciencia del siguiente modo: "Ahora considero que puedo prescindir de ciertas cosas que no son tan necesarias". Su afirmación contiene el sentido del presente ("ahora"), como una circunstancia personal distinta a la pasada, sobre la cual tiene la posibilidad de discriminar lo que valora $\mathrm{u}$ origina bienestar de aquello que no lo logra.

Esta categoría expone dos ángulos de la conciencia de la temporalidad, tal como emergió entre los entrevistados. Por una parte, que la experiencia de bienestar no es un continuo, sino que es vivenciada en lapsos, con mayor o menor incidencia en el bienestar, y que estos lapsos componen la percepción general de bienestar. Un entrevistado lo ejemplifica al decir: "No considero que el bienestar sea permanente sino por momentos".

Las experiencias de bienestar pueden acompañarse de otras opuestas, como la culpa o el arrepentimiento, que pueden ocurrir luego de haber comprado un producto buscando una satisfacción, pero que tiempo después manifiesta su incapacidad de atender del todo la necesidad y sostener la experiencia de satisfacción. Uno de los jóvenes describe esta vivencia: "Ahora me voy a Starbucks, pero en la tarde voy a estar pensando "por qué gasté tanto, mejor hubiera comprado un menú, piensas si vale o no vale la pena". Por su parte, otra entrevistada dice: "Ahora que ya no lo uso, y no me parece la gran cosa, me arrepiento de haberlo comprado, porque no lo uso y ya no hay un bienestar".

La categoría descubre la conciencia entre los entrevistados de que el bienestar no es una experiencia absoluta, y que el consumo o tenencia de bienes no necesariamente lo origina, y que es susceptible de no poder sostenerlo en el tiempo.

\section{Categoría 5: La pertenencia}

Esta categoría describe la función instrumental del consumo para atender la necesidad de pertenencia y aceptación por parte de un grupo entre los jóvenes. Se pone de manifiesto que las prácticas de consumo, o la posesión de marcas de marcas determinadas, son utilizadas por los participantes como un vehículo para satisfacer su necesidad de pertenencia y aceptación. La naturaleza social humana, y la necesidad de 
pertenecer a un grupo que acoja al individuo y le ofrezca seguridad, se encuentran en las realidades expresadas por los entrevistados.

Por ejemplo, un entrevistado lo expresa del siguiente modo: "Si ves que todos tus amigos tienen una marca también la quieres tener por un sentido de pertenencia". Otro entrevistado dice: "[uno] está en un entorno en el cual siente una presión de tener cierto producto, busca pertenencia". Un producto o marca parecen servir el propósito de elemento en común, que llama hacia sí al grupo humano y le otorga homogeneidad.

La misma experiencia la expresa una entrevistada cuando narra: "Siempre veo que mis amigas tienen carteras de marca, [si la mía es igual] ya no tendría vergüenza dejar mi cartera en la silla e ir a conversar, siento que hablarán bien de mí, sobre todo si se trata de estar en una fiesta con tu círculo social".

La pertenencia y aceptación aparecen como necesidades sociales relevantes para la experiencia de bienestar. A su vez, trascienden la dinámica de consumo y la incidencia de los mensajes de las marcas, dando lugar a vivencias de bienestar fundadas en la socialización y no en las características funcionales de un bien. Por ejemplo, la misma participante, luego de afirmar de que consumir una popular marca de bebidas gasificadas no le origina la felicidad que comunica en su publicidad, menciona que sí obtiene una experiencia de felicidad cuando miembros de su grupo de amigos perciben positivamente el consumo de dicha marca de bebida gasificada: "creo que es una felici- dad indirecta, la felicidad de sentirte aceptado por un grupo".

Una experiencia similar es narrada por otro entrevistado, quien señala cómo el consumo en una cadena de comida rápida, de una marca con alta recordación, es menos relevante que el hecho de compartir momentos con su grupo de amigos en un local de dicha marca. Lo narra de la siguiente manera: "el objetivo no es la comida, sino el compartir, estar con ellos. No importa qué estés comiendo tampoco, porque puede ser que no te gusta mucho esa comida, pero igual vas a ir".

La necesidad de que el grupo valide el consumo también aparece como un requisito de la necesidad de pertenencia y aceptación. Se observa cuando la satisfacción de la compra de un producto necesita ser confirmada por parte de aquellos a quienes se les exhibe, o se espera la evidencia de algún tipo de reacción positiva ante la exhibición de lo comprado. Uno de los entrevistados lo verbaliza del siguiente modo: "otras [jóvenes] saben lo que tienen, y también quieren que las demás personas sepan lo que tienen, sino sienten que compraron algo por las puras, porque la gente no se lo va a aplaudir".

Atender la necesidad social de pertenencia se presenta como una realidad relevante para los jóvenes. El consumo de productos o servicios, así como las marcas vinculadas, se presentan como conductos válidos para servir esta necesidad, y asegurar la experiencia de bienestar que se origina de la interrelación del joven con los grupos a los que pertenece, o de aquellos grupos que le sirven de referencia. 


\section{Discusión}

Las investigaciones cualitativas buscan comprender los fenómenos desde la perspectiva de los participantes (Creswell, 2013; Gentles, Ploeg y McKibbon, 2015), y no tiene por objeto obtener resultados generalizables, como si es lo propio de los estudios cuantitativos. Este estudio procura conocer el modo en que jóvenes universitarios perciben que el consumo y las marcas incide en sus experiencias de bienestar subjetivo y las de sus pares.

El abordaje del tema se apoya en lo reportado en investigaciones previas de que el proceso y acto de consumo incide en los niveles de bienestar subjetivo de las personas (Pirouz, 2008; Tov y Diener, 2013; Diener y Biswas-Diener, 2002), pero que a su vez este efecto tiene limitaciones en el sostenimiento de la experiencia de bienestar (Iyer y Muncy, 2016; Kay y Jost 2003; Sengupta et al., 2012; Hoffmann y Lee, 2016; Dittmar, Bond, Hurst y Kasser, 2014), y que las diferencias individuales modulan la vivencia de bienestar en cada persona. (Oishi, Suh y Lucas, 1999)

La relación entre consumo y bienestar se observó en las categorías que emergieron del análisis realizado. Si bien el consumo de marcas se halla presente de modo transversal, su participación sobre el bienestar subjetivo no parece ocurrir de una única manera entre los participantes. Por el contrario, y acorde con lo observado por Oishi, Suh y Lucas (1999), entre ellos se advierten características y valoraciones distintas cuando se refieren a esta experiencia.
En efecto, la categoría titulada 'Relativo a cada uno' da particular cuenta de este hallazgo. La categoría describe la amplitud de ángulos y matices con que los participantes abordan la búsqueda de bienestar en el consumo de productos. Cada persona presenta distintas aproximaciones al proceso de compra, y también una gradación valorativa hacia las marcas de categorías que mencionaron. Es decir que, lejos de ser una experiencia absoluta, se trata de una experiencia dependiente de cada individuo.

Un factor que interviene en la experiencia de bienestar es el marco valorativo que cada individuo tiene hacia el consumo y las marcas. Este hecho es congruente con el trabajo de Crawford et al. (2000), quienes sostienen que la influencia de lo material sobre el bienestar subjetivo se encuentra mediado por el grado de deseos materiales de los sujetos. Se advierte que este marco valorativo es originado por aprendizaje de los padres, quienes, en su rol educativo, y por sus propias acciones de consumo, han influido en la mentalidad y los criterios de estos jóvenes. De esta manera, el entorno familiar personaliza los parámetros de valoración y, en consecuencia, su estilo de relacionarse y los grados de apego de los jóvenes hacia los bienes materiales y las marcas.

Si bien los entrevistados pertenecen a un nivel socioeconómico medio y superior, con lo cual el acceso a productos y marcas específicas es una realidad viable, entre ellos se observa la conciencia de que dicho acceso no es ilimitado y que sus padres deben trabajar para obtener los fondos para financiarlos. La 
circunstancia económica y el sentido de valor del consumo explican el hecho de que algunos participantes tengan conciencia de que pueden prescindir de ciertos bienes, incluyendo las marcas. En particular entre aquellos cuyo marco valorativo evidencia un menor apego materialista, entrevistados quienes no comprenden que los bienes sean determinantes del bienestar. Esta apreciación respecto al consumo es congruente con lo señalado por Armenta, et al. (2014), Buechel, Townsend y Moreau (2018) y Hovasapian y Levine (2018) referente a la adaptación hedónica. Hay conciencia de que la experiencia originada por el consumo puede ser limitada o relativa sobre el bienestar general.

Por otra parte, es interesante notar que, con independencia de la naturaleza y variedad de necesidades que cada individuo tiene, es su satisfacción lo que pronostican que da origen a la experiencia de bienestar. Es decir, cuando no falta aquello consideran que deben poseer o alcanzar. Esta observación está alineada con los hallazgos de distintos estudios donde también se analiza el bienestar y la necesidad. (Tov y Diener, 2013; Verma, 2017; Dolan, Peasgood y White, 2008)

Como ya se ha discutido, la relevancia en la posesión de marcas o el apego a lo material no es una realidad homogénea entre los participantes, con lo cual no se presentan como requisitos declarados de bienestar. El que no sean un requisito debe comprenderse en el contexto de que los entrevistados pertenecen a un nivel socioeconómico medio y superior, con lo cual el acceso a productos y marcas específicas es una realidad que se da por supuesta. Es probable que, en un escenario de escasez, los mismos participantes manifiesten que lo material sí es requisito de bienestar. En cualquier caso, se evidenciaría nuevamente que la satisfacción de la necesidad es condición básica de bienestar.

A pesar del carácter individual de la experiencia de bienestar, la pertenencia social aparece como una necesidad común entre los participantes, quienes procuran atender esta necesidad con el consumo de marcas específicas. La pertenencia se refiere a la necesidad de afiliarse y ser aceptado por los miembros de un grupo (Leary y Baumeister, 2017), los cuales pueden ser de distinta naturaleza, por ejemplo, un grupo de compañeros de estudio, los compañeros de trabajo, o de un equipo deportivo o de voluntariado. La necesidad de pertenencia va más allá de conocer a los otros miembros del grupo, sino también obtener su aceptación y apoyo.

Ante esta necesidad, los jóvenes parecen utilizar la marca como un conducto para alcanzar y sostener la pertenencia y aceptación entre sus pares. La declaración de un entrevistado es ilustrativa: "Si ves que todos tus amigos tienen una marca también la quieres tener por un sentido de pertenencia". Esta categoría emergió debido a que los jóvenes encuentran en los productos y las marcas un instrumento de identidad y estatus, elementos que sirven de código en la dinámica de socialización. De esta manera, un producto con marca establecida opera a modo de clave de pertenencia, como 
una señal de que quien la posee puede pertenecer a un grupo, o de tener algo en común entre otros pares. Se trata de una mecánica circular, pues cada individuo que usa una marca la constituye en elemento de homogenización del grupo, y le otorga sentido como medio para la aceptación y pertenencia. Esta realidad se debe originar cuando varios de los miembros de un grupo dan un mismo sentido a la marca o su utilización, tal como lo explica el marco conceptual denominado Sistema de Marcas de Luedicke (2006).

Luedicke (2006) señala que la marcas tienen un significado dentro de cada cultura particular, el cual permite que las personas procesen los mensajes de la marca, tales como su publicidad, o las historias de amigos referidas al producto, con un cierto grado de homogeneidad, así como dar un sentido simbólico a su uso (Bettingen y Luedicke, 2009; Luedicke y Giesler, 2007). En el Sistema de Marcas, el autor y sus colegas sostienen que estos significados evolucionan a través de la comunicación y las señales de cada marca, como sus colores más representativos, logotipos o imágenes que facilitan su recordación, y hasta sonidos u olores (Giesler, 2003; Luedicke, 2006). De acuerdo con sus hallazgos, el bienestar puede resultar de las experiencias de los consumidores con estas guías sensoriales de marca, y el sentido social que las marcas adquieren. La propuesta de Luedicke (2006) y Bettingen y Luedicke (2009) es que el consumo de una marca tiene el potencial de originar bienestar en función al sentido positivo que la sociedad le otor- gue a esa marca. Por ejemplo, un reloj o auto de una marca costosa tiene asociados una serie de guías de marca que en una sociedad comunican estatus, con lo cual su consumo puede ser un conducto para la experiencia de bienestar originada por la apreciación de los miembros de esa cultura. El Sistema de Marcas ofrece un marco conceptual adecuado para observar el significado cultural de las marcas, su consumo y relación con el bienestar subjetivo.

La presente investigación tiene una serie de limitaciones. Por una parte, todos los participantes manifestaron que sus padres cubren sus necesidades económicas, sin embargo, podrían hacerlo con distintos grados de holgura. Con lo cual, los hallazgos podrían tener variaciones en caso los sujetos tuvieran mayor comodidad financiera. Igual ocurriría en el caso contrario, si el estudio se realizara con participantes jóvenes inmersos en un escenario de escasez material. Futuras investigaciones podrán fijar un rango de disponibilidad monetaria como parte de los criterios de inclusión, y examinar la experiencia en dichos casos. Igualmente, próximas investigaciones pueden indagar con jóvenes independizados económicamente de sus padres. Por otro lado, en la selección de participantes no se consideró la personalidad como una variable de análisis, por lo cual no es posible observar, por ejemplo, si las prácticas de consumo o la valoración de las marcas, dan lugar a experiencias de bienestar distintas entre individuos cuya personalidad les permite mayor grado de influencia sobre sus pares del grupo. Futuras 
investigaciones podrán incorporar esta variable psicográfica. En ambos casos, los resultados limitan la transferibilidad de los resultados hacia la comprensión de otras cohortes de jóvenes.

La pertenencia fue una categoría emergente de este estudio. Investigaciones posteriores pueden abordar, con mayor detalle y profundidad, las prácticas de consumo destinadas a lograr la pertenencia al grupo, así como los elementos que componen el sentido de pertenencia entre los jóvenes cuando es mediada por el consumo o las marcas. Asimismo, también cabe estudiar si la pertenencia al grupo no es, precisamente, un objetivo que la marca propone a sus consumidores entre sus mensajes publicitarios. A ello se añade que en este estudio exploratorio se han utilizado de forma indistinta la pertenencia al grupo y la aceptación por parte del grupo. Parece conveniente que investigaciones futuras analicen posibles diferencias en cada caso, o si ambas realidades pueden tratarse de modo intercambiable ante el consumo como conducto de bienestar. En esta misma línea, también cabe que otras investigaciones indaguen si la conciencia de encajar, en un grupo o con el perfil de un grupo, también es un objetivo que los jóvenes atienden instrumentalizando el consumo.

Profesionales de las comunicaciones de marketing, así como responsables de la gestión de marcas en las empresas, pueden tomar en consideración el sentido instrumental de los productos en la socialización de las personas. Esta información enriquece la precisión del perfil de sus consumidores, así como del posi- cionamiento y las propuestas de valor de cada marca. En ambos casos puede ser de utilidad para la planificación y gestión de la marca, así como el diseño de estrategias de comunicación.

A lo largo del estudio no se manifestó una presencia consciente de la publicidad en los discursos de los jóvenes entrevistados. Dicha ausencia no significa que las comunicaciones de marca carezcan de influencia sobre el consumo o el deseo de marcas; por el contrario, puede significar que su influencia ocurre en un nivel pre-atento, y que su indagación puede ser objeto de próximas investigaciones.

\section{Conclusiones}

Con base en los resultados del análisis cualitativo y de alcance exploratorio, se advierte que los jóvenes participantes perciben un vínculo entre el consumo y el bienestar subjetivo. Esta experiencia de consumo-bienestar aparece modulada por distintos factores de la persona y su entorno, en particular su sentido de necesidad material formado desde su entorno de crecimiento. Con lo cual la forma final de sus creencias sobre el bienestar y el consumo es relativo a cada individuo.

También se observa que los jóvenes perciben que la incidencia del consumo y las marcas sobre el bienestar subjetivo ocurre de forma mediada. Es decir, los jóvenes encuentran en los actos de compra, y aquellas marcas que sus pares valoran, un conducto para asistir a sus vínculos grupales. Ante este fenómeno el bienestar expe- 
rimentado no parece desprenderse de las características funcionales del bien, sino a la experiencia de aceptación y pertenencia que el consumo del bien ocasiona. El consumo y marcas como conductos para la pertenencia también se presentan como realidad relativa a cada individuo, de su valoración material aprendida y su propia forma de ser.

En cuanto a los elementos que consi- deran en las conductas de consumo orientadas a experimentar bienestar, en los resultados se advierte que las señas de marca son relevantes, debido a que permiten trasmitir rasgos de la identidad personal, o de la imagen que se desea proyectar. Los participantes evidencian utilizar las señas como guías para orientar a sus pares, o miembros de grupos, en las conductas de socialización y pertenencia.

\section{Bibliografía}

Al Mandil, K. (2016). Modelling the relationship between brand experience dimensions and the antecedents of happiness within the context of dining services (Tesis doctoral). Brunel University. Londres, Reino Unido.

Arias, R., \& Otnes, C. (2017). The Belonging Process: Elements and Enactment Within a Consumption Context. ACR North American Advances, 45, 500-502. Ayelet Gneezy, Vladas Griskevicius, \& Patti Williams (Eds.), Duluth, MN: Association for Consumer Research.

Arias, R., \& Otnes, C. (2006). Illuminating consumers' proactive pursuits of the sense of belonging. En Robert Mittelstaedt doctoral symposium proceedings. University of Nebraska Lincoln. (pp. 23-38).

Armenta, C., Bao, K. J., Lyubomirsky, S., \& Sheldon, K. M. (2014). Is lasting change possible? Lessons from the Hedonic Adaptation Prevention model. In Stability of Happiness: Theories and evidence on whether happiness can change. 57-74. Sheldon, K. M., \& Lucas, R. E. (Eds.) Elsevier.

Bengtsson, M. (2016). How to plan and perform a qualitative study using content analysis. NursingPlus Open, 2, 8-14. https://doi.org/10.1016/J.NPLS.2016.01.001

Bettingen, J. F., \& Luedicke, M. K. (2009). Can brands make us happy? A research framework for the study of brands and their effects on happiness. ACR North American Advances. 36, 308-318. Ann L. McGill \& Sharon Shavitt (Eds.), Duluth, MN: Association for Consumer Research.

Briñol, P., Rucker, D. D., \& Petty, R. E. (2015). Naïve theories about persuasion: Implications for information processing and consumer attitude change. International Journal of Advertising, 34(1), 85-106.

Brülde, B. (2014) 'Hedonism', in Michalos, A.C. (ed.) Encyclopedia of Quality of Life and WellBeing Research. New York: Springer, pp. 2837-2842.

Buechel, E. C., Townsend, C., \& Moreau, P. (2018). Buying Beauty for the Long Run: (Mis) predicting Liking of Product Aesthetics. Journal of Consumer Research.

Busseri, M. A., \& Sadava, S. W. (2011). A review of the tripartite structure of subjective wellbeing: Implications for conceptualization, operationalization, analysis, and synthesis. Personality and Social Psychology Review, 15(3), 290-314. 
Caprariello, P. A., \& Reis, H. T. (2013). To do, to have, or to share? Valuing experiences over material possessions depends on the involvement of others. Journal of Personality and Social Psychology, 104(2), 199-215.

Carter, T. J., \& Gilovich, T. (2010). The relative relativity of material and experiential purchases. Journal of Personality and Social Psychology, 98(1), 146-159.

Codina, J. N. B., Rodríguez, P. G., \& Cadena, P. V. (2017). La economía de la experiencia y el marketing emocional: estrategias contemporáneas de comercialización. Daena: International Journal of Good Conscience, 12(2), 159-170.

Coyne, I. T. (1997). Sampling in qualitative research. Purposeful and theoretical sampling; merging or clear boundaries? Journal of advanced nursing, 26(3), 623-630.

Creswell, J. W. (2013). Qualitative inquiry and research design: choosing among five approaches. California: Sage.

Crouch, M., \& McKenzie, H. (2006). The logic of small samples in interview-based qualitative research. Social science information, 45(4), 483-499.

Chancellor, J. \& Lyubomirsky, S. (2014). Money for Happiness: The Hedonic Benefits of Thrift. In Consumption and Well-Being in the Material World, (Ed) Mariam Tatzel (13-48). New York: Springer.

Daymon, C., \& Holloway, I. (2010). Qualitative research methods in public relations and marketing communications. Routledge.

Diener, E. (1994). Assessing subjective well-being: Progress and opportunities. Social indicators research, 31(2), 103-157.

Diener, E., \& Biswas-Diener, R. (2002). Will money increase subjective well-being? Social indicators research, 57(2), 119-169.

Diener, E., Scollon, C. N., \& Lucas, R. E. (2009). The Evolving Concept of Subjective Well-Being: The Multifaceted Nature of Happiness. In: Diener E. (eds) Assessing Well-Being. Social Indicators Research Series, vol 39. Dordrecht: Springer.

Diener, E., Heintzelman, S. J., Kushlev, K., Tay, L., Wirtz, D., Lutes, L. D., \& Oishi, S. (2017). Findings all psychologists should know from the new science on subjective well-being. Canadian Psychology/psychologie canadienne, 58(2), 87.

Dittmar, H., Bond, R., Hurst, M., \& Kasser, T. (2014). The relationship between materialism and personal well-being: A meta-analysis. Journal of personality and social psychology, 107(5), 879.

Dolan, P., Peasgood, T., \& White, M. (2008). Do we really know what makes us happy? A review of the economic literature on the factors associated with subjective well-being. Journal of economic psychology, 29(1), 94-122.

Englander, M. (2012). The interview: Data collection in descriptive phenomenological human scientific research. Journal of phenomenological psychology, 43(1), 13-35.

El Hedhli, K., Chebat, J. C., \& Sirgy, M. J. (2013). Shopping well-being at the mall: Construct, antecedents, and consequences. Journal of Business Research, 66(7), 856-863.

Fenzl, T., \& Philipp, M. (2013). QCAmap: eine interaktive Webapplikation für Qualitative Inhaltsanalyse. En 15th Biennial EARLI Conference for Research on Learning and Instruction (pp. 333-339). Munich. 
Fernández, A. R., \& Goñi-Grandmontagne, A. (2011). La estructura tridimensional del bienestar subjetivo. Anales de psicología, 27(2), 327.

Fusch, P. I., \& Ness, L. R. (2015). Are we there yet? Data saturation in qualitative research. The qualitative report, 20(9), 1408-1416.

Gentles, S. J., Charles, C., Ploeg, J., \& McKibbon, K. (2015). Sampling in qualitative research: Insights from an overview of the methods literature. The Qualitative Report, 20(11), 1772-1789.

Giesler, M. (2003). Social Systems in Marketing. European Advances in Consumer Research, Vol. 6, eds. Darach Turley and Stephen W. Brown, Valdosta, GA: Association for Consumer Research, 249-256.

Giesler, M., \& Venkatesh, A. (2005). Reframing the Embodied Consumer As Cyborg a Posthumanist Epistemology of Consumption. In NA - Advances in Consumer Research Volume 32, eds. Geeta Menon and Akshay R. Rao, Duluth, MN: Association for Consumer Research, Pages: 661-669.

Goulding, C. (2005). Grounded theory, ethnography and phenomenology: A comparative analysis of three qualitative strategies for marketing research. European journal of Marketing, 39(3/4), 294-308.

Graneheim, U. H., \& Lundman, B. (2004). Qualitative content analysis in nursing research: concepts, procedures and measures to achieve trustworthiness. Nurse education today, 24(2), 105-112.

Guest, G., Bunce, A., \& Johnson, L. (2006). How many interviews are enough? An experiment with data saturation and variability. Field Methods, 18(1), 59-82.

Guevarra, D. A., \& Howell, R. T. (2015). To have in order to do: Exploring the effects of consuming experiential products on well-being. Journal of Consumer Psychology, 25(1), 28-41.

Hoffmann, S., \& Lee, M. S. (2016). Consume Less and Be Happy? Consume Less to Be Happy! An Introduction to the Special Issue on Anti-Consumption and Consumer WellBeing. Journal of Consumer Affairs, 50(1), 3-17.

Howell, R. T., \& Howell, C. J. (2008). The relation of economic status to subjective well-being in developing countries: A meta-analysis. Psychological bulletin, 134(4), 536.

Howell, R. T., \& Hill, G. (2009). The mediators of experiential purchases: Determining the impact of psychological needs satisfaction and social comparison. The Journal of Positive Psychology, 4(6), 511-522.

Howell, R. T., Rodzon, K. S., Kurai, M., \& Sanchez, A. H. (2010). A validation of well-being and happiness surveys for administration via the Internet. Behavior research methods, 42(3), 775-784.

Hovasapian, A., \& Levine, L. J. (2018). Keeping the magic alive: social sharing of positive life experiences sustains happiness. Cognition and Emotion, 1-12.

Iyer, R., \& Muncy, J. A. (2016). Attitude toward Consumption and Subjective Well-Being. Journal of Consumer Affairs, 50(1), 48-67.

Joshi, A., \& Nema, G. (2015). Emotional branding: A new way to create brand loyalty for FMCG companies. Journal of Management Value and Ethics, 5(2). 
Kahn, R. L., \& Juster, F. T. (2002). Well-Being: concepts and measures. Journal of Social Issues, 58(4), 627-644.

Kahneman, D. (1999). Objective happiness. Well-being: The foundations of hedonic psychology, 3 , 25.

Kahneman, D., Krueger, A., Schkade, D. A., Schwarz, N., \& Stone, A. A. (2004). A survey method for characterizing daily life experience: The day reconstruction method. Science, 306(5702), 1776-80.

Kahneman, D., \& Krueger, A. B. (2006). Developments in the measurement of subjective well-being. The Journal of Economic Perspectives, 20(1), 3-24.

Kahneman, D., Kahneman, D., \& Tversky, A. (2003). Experienced utility and objective happiness: A moment-based approach. The psychology of economic decisions, 1, 187-208.

Kay, A. C., \& Jost, J. T. (2003). Complementary justice: effects of " poor but happy" and” poor but honest" stereotype exemplars on system justification and implicit activation of the justice motive. Journal of personality and social psychology, 85(5), 823.

Kim-Prieto, C., Diener, E., Tamir, M., Scollon, C., \& Diener, M. (2005). Integrating the diverse definitions of happiness: A time-sequential framework of subjective well-being. Journal of Happiness Studies, 6(3), 261-300.

Kushlev, K., Heintzelman, S. J., Lutes, L. D., Wirtz, D., Oishi, S., \& Diener, E. (2017). Enhance: Design and rationale of a randomized controlled trial for promoting enduring happiness \& well-being. Contemporary clinical trials, 52, 62-74.

Leary, M. R., \& Baumeister, R. F. (2017). The need to belong: Desire for interpersonal attachments as a fundamental human motivation. In Interpersonal Development (pp. 57-89).

Linley, P. A., Joseph, S., Harrington, S., \& Wood, A. M. (2006). Positive psychology: Past, present, and (possible) future. The Journal of Positive Psychology, 1(1), 3-16.

Luedicke, M. (2006). A theory of marketing: Outline of a social systems perspective. Bieswaden: Deutscher Universitats-Verlag.

Luedicke, M., \& Giesler, M. (2007). Brand communities and their social antagonists: insights from the Hummer case. Consumer Tribes, 275-95.

Luedicke, M., \& Giesler, M. (2005). Brand Systems: a Conceptual Framework For the Sociological Analysis of Brand Phenomena, in E - European Advances in Consumer Research Volume 7, eds. Karin M. Ekstrom and Helene Brembeck, Goteborg, Sweden: Association for Consumer Research, pp., 520-521.

Mead, N. L., Baumeister, R. F., Stillman, T. F., Rawn, C. D., \& Vohs, K. D. (2011). Social Exclusion Causes People to Spend and Consume Strategically in the Service of Affiliation. Journal of Consumer Research, 37(5), 902-919.

Morse J.M. (1991) Strategies for sampling. En Qualitative Nursing Research: A Contemporary Dialogue, ed. J. M. Morse, Newbury Park, California: Sage, pp. 127-145.

Muniz, A. M., \& O'Guinn, T. C. (2001). Brand community. Journal of Consumer Research, 27(4), 412-432.

Ness, P., \& Fusch, L. (2015). Are We There Yet? Data Saturation in Qualitative Research. The Qualitative Report, 20(9), 1409-1416. 
Nickerson, C., Schwarz, N., \& Diener, E. (2007). Financial aspirations, financial success, and overall life satisfaction: who? and how? Journal of Happiness Studies, 8(4), 467-515.

Oishi, S., \& Schimmack, U. (2010). Residential mobility, well-being, and mortality. Journal of personality and social psychology, 98(6), 980.

Palinkas, L. A., Horwitz, S. M., Green, C. A., Wisdom, J. P., Duan, N., \& Hoagwood, K. (2015). Purposeful sampling for qualitative data collection and analysis in mixed method implementation research. Administration and Policy in Mental Health and Mental Health Services Research, 42(5), 533-544.

Patton, M. Q. (2002). Qualitative research and evaluation methods (3rd ed.). Thousand Oaks, CA: Sage.

Pirouz, D. (2008). The Subjective Well-Being of Buying: a Grounded Theory Analysis of Habitual Acts of Consumption. Advances in Consumer Research - ACR North American Advances.

Reynolds, F., Vivat, B., \& Prior, S. (2008). Women's experiences of increasing subjective wellbeing in CFS/ME through leisure-based arts and crafts activities: A qualitative study. Disability and Rehabilitation, 30(17), 1279-1288.

Ryan, R. M., \& Deci, E. L. (2001). On happiness and human potentials: A review of research on hedonic and eudaimonic well-being. Annual review of psychology, 52(1), 141-166.

Saldana, J. (2013). The coding manual for qualitative researchers. London: Sage.

Saunders, B., Sim, J., Kingstone, T., Baker, S., Waterfield, J., Bartlam, B., ... \& Jinks, C. (2018). Saturation in qualitative research: exploring its conceptualization and operationalization. Quality E Quantity, 52(4), 1893-1907.

Seligman, M. E., \& Csikszentmihalyi, M. (2014). Positive psychology: An introduction. In Flow and the foundations of positive psychology (pp. 279-298). Springer Netherlands.

Sengupta, N. K., Osborne, D., Houkamau, C. A., Hoverd, W. J., Wilson, M. S., Halliday, L., \& Sibley, C. G. (2012). How much happiness does money buy? Income and subjective wellbeing in New Zealand. New Zealand Journal of Psychology, 41(2), 21-34.

Schor, J. B. (1999). The overspent American: Why we want what we don't need. New York: HarperPerennial.

Stone, A. A., \& Mackie, C. D. (Eds.). (2013). Subjective Well-Being : measuring happiness, suffering, and other dimensions of experience. Panel on Measuring Subjective Well-Being in a PolicyRelevant Framework (p. 188). Washington, DC: Comittee on Nations Statistics. The National Academy Press.

Titchen, A., \& Hobson, D. (2011). Understanding Phenomenology through Reverse Perspectives. In B. Somekh \& C. Lewin (Eds.), Theory and methods in social research $\left(2^{\circ}\right)$. Sage.

Tkach, C., \& Lyubomirsky, S. (2006). How do people pursue happiness?: Relating personality, happiness-increasing strategies, and well-being. Journal of happiness studies, 7(2), 183-225.

Tov, W. \& Diener, E. (2013). Subjective Well-Being. En: Keith, K. D. (Ed.). (2013). The encyclopedia of cross-cultural psychology. Wiley-Blackwell. 
Verma, R. (2017). Gross National Happiness: meaning, measure and degrowth in a living development alternative. Journal of Political Ecology, 24(1), 476-490.

Yin, R. K. (2011). Qualitative research from start to finish. New York, NY: Guilford Press. 